\title{
Dense chaos for continuous interval maps
}

\author{
Sylvie Ruette
}

March 8, 2005.

\begin{abstract}
A continuous map $f$ from a compact interval $I$ into itself is densely (resp. generically) chaotic if the set of points $(x, y)$ such that $\limsup _{n \rightarrow+\infty}\left|f^{n}(x)-f^{n}(y)\right|>0$ and $\liminf _{n \rightarrow+\infty}\left|f^{n}(x)-f^{n}(y)\right|=0$ is dense (resp. residual) in $I \times I$. We prove that if the interval map $f$ is densely but not generically chaotic then there is a descending sequence of invariant intervals, each of which containing a horseshoe for $f^{2}$. It implies that every densely chaotic interval map is of type at most 6 for Sharkovskil's order (that is, there exists a periodic point of period 6 ), and its topological entropy is at least $\log 2 / 2$. We show that equalities can be realised.
\end{abstract}

\section{Introduction}

This article deals with the dynamics of interval maps, that is, continuous maps $f: I \rightarrow I$ where $I$ is a compact interval in $\mathbb{R}$. We first give some notations used in this paper. An invariant set (for the map $f$ ) is a closed non empty subset $A$ such that $f(A) \subset A$. A transitive subset (for $f$ ) is an invariant set $A$ such that $\left.f\right|_{A}$ is transitive (see, e.g., [6] for the definition of transitivity). The length of an interval $J$ is denoted by $|J|$. An interval $J$ is non degenerate if $|J|>0$, that is, $J$ is neither empty nor reduced to a single point.

In [10] Li and Yorke called chaotic some kind of behaviour of interval maps, although without formal definition. The following notions of Li-Yorke pairs and Li-Yorke chaos were derived from this article.

Definition 1.1 Let $T: X \rightarrow X$ be a continuous map on a metric space $X$. If $x, y \in X$ and $\delta>0,(x, y)$ is called a Li-Yorke pair of modulus $\delta$ if

$$
\limsup _{n \rightarrow+\infty} d\left(T^{n}(x), T^{n}(y)\right)>\delta \quad \text { and } \quad \liminf _{n \rightarrow+\infty} d\left(T^{n}(x), T^{n}(y)\right)=0 ;
$$

$(x, y)$ is a Li-Yorke pair if it is a Li-Yorke pair of modulus $\delta$ for some $\delta>0$. The set of Li-Yorke pairs of modulus $\delta$ is denoted by $\operatorname{LY}(T, \delta)$ and the set of Li-Yorke pairs by $\operatorname{LY}(T)$.

Definition 1.2 Let $T: X \rightarrow X$ be a continuous map on a metric space $X$. The system $(X, T)$ is said chaotic in the sense of Li-Yorke if there exists an uncountable set $S \subset X$ such that for all $x, y \in S, x \neq y,(x, y)$ is a Li-Yorke pair.

The definition of generic chaos is due to Lasota (see [13]). Being inspired by this definition, Snoha defined generic $\delta$-chaos, dense chaos and dense $\delta$-chaos [16].

Definition 1.3 Let $T: X \rightarrow X$ be a continuous map on a metric space $X$ and $\delta>0$.

2000 Mathematics Subject Classification. 37E05, 37B40.

Nonlinearity, 18, 1691-1698, 2005. 
- $T$ is generically chaotic if $\operatorname{LY}(T)$ is residual in $X^{2}$,

- $T$ is generically $\delta$-chaotic if $\operatorname{LY}(T, \delta)$ is residual in $X^{2}$,

- $T$ is densely chaotic if $\operatorname{LY}(T)$ is dense in $X^{2}$,

- $T$ is densely $\delta$-chaotic if $\operatorname{LY}(T, \delta)$ is dense in $X^{2}$.

Generic $\delta$-chaos obviously implies both generic chaos and dense $\delta$-chaos, which in turn imply dense chaos.

In [16], Snoha proved that for an interval map generic chaos implies generic $\delta$-chaos for some $\delta>0$ and the notions of generic $\delta$-chaos and dense $\delta$-chaos coincide, but a densely chaotic interval map may be not generically chaotic. In [17] Snoha gave a characterisation of densely chaotic interval maps and proved that for piecewise monotone interval maps the notions of dense chaos and generic chaos coincide. He asked what the infimum of topological entropy and the type for Sharkovskiı̌'s order are for densely chaotic interval maps. We recall Sharkovskiıı's theorem (see [18]):

Theorem 1.4 (Sharkovskiu[14]) Consider the following order:

$$
3 \triangleleft 5 \triangleleft 7 \triangleleft 9 \triangleleft \cdots \triangleleft 2 \cdot 3 \triangleleft 2 \cdot 5 \triangleleft 2 \cdot 7 \triangleleft \cdots \triangleleft 2^{2} \cdot 3 \triangleleft 2^{3} \cdot 5 \triangleleft \cdots \triangleleft 2^{n} \triangleleft \cdots 2^{3} \triangleleft 2^{2} \triangleleft 2 \triangleleft 1
$$

If the interval map $f$ has a periodic point of period $n$ then it has periodic points of period $m$ for all $m \triangleright n$.

According to Theorem 1.4, the set of periods of periodic points of an interval map $f$ is, either $\{m \mid m \unrhd n\}$ for some positive integer $n$, and in this case $f$ is said of type $n$, or $\left\{2^{n} \mid n \geq 0\right\}$, and $f$ is said of type $2^{\infty}$. Remark that there exist interval maps of all types [18, 5].

Our first motivation was to answer Snoha's questions. In Theorem 3.1, we actually obtain a result on the structure of interval maps that are densely chaotic but not generically chaotic: for such a map $f$, there exists a descending sequence of invariant intervals, with lengths tending to 0 , and each of them contains a horseshoe for the map $f^{2}$ (that is, two closed non degenerate intervals $J, K$ with disjoint interiors such that $\left.f^{2}(J) \cap f^{2}(K) \supset J \cup K\right)$. On the other hand, Snoha gave a characterisation of generic chaos in term of transitive subintervals:

Theorem 1.5 (Snoha [16]) Let $f$ be an interval map. The following conditions are equivalent:

- $f$ is generically chaotic,

- either there exists a unique transitive non degenerate subinterval, or there exist two transitive non degenerate subintervals with a common endpoint. Moreover for every non degenerate interval $J, f^{n}(J)$ is non degenerate and there exist a transitive subinterval $T$ and an integer $n \geq 0$ such that $f^{n}(J) \cap \operatorname{Int}(T) \neq \emptyset$.

We deduce from the structure of densely chaotic interval maps that such maps are at most of type 6 for Sharkovskiı̌'s order and their topological entropy is greater than or equal to $\frac{\log 2}{2}$ (Corollary 3.6). Example 3.7 shows that equalities are possible, and in addition they can be realised by generically chaotic interval maps.

In [11] Murinová generalised Snoha's work and showed that for a complete metric space $X$, generic $\delta$-chaos and dense $\delta$-chaos are equivalent. She also exhibited a generically chaotic system which is not generically $\delta$-chaotic for any $\delta>0$. 
If $X$ is a complete metric space and $G \subset X \times X$ is a dense $G_{\delta}$-set then using Kuratowski's theorem (see, e.g., 12]) one can find an uncountable set $S$ such that $S \times S$ deprived of the diagonal of $X \times X$ is included in $G$ (see e.g., [7, Lemma 3.1]). Therefore a generically chaotic map on a complete metric space is chaotic in the sense of Li-Yorke. In [8], Kuchta and Smítal showed that on the interval the existence of one Li-Yorke pair is enough to imply chaos in the sense of Li-Yorke, consequently dense chaos implies Li-Yorke chaos for interval maps. However it is not known whether dense chaos implies Li-Yorke chaos in general.

Section 3 contains our main results. Some preliminary lemmas are needed, they are stated in Section 2

\section{Preliminary results}

Lemma 2.1 Let $f$ be a densely chaotic interval map.

1. If $J$ is a non degenerate interval then $f^{n}(J)$ is non degenerate for all $n \geq 0$.

2. Consider disjoint non degenerate intervals $J_{1}, \ldots, J_{p}$ such that $f\left(J_{i}\right) \subset J_{i+1} \bmod p$. Then either $p=1$, or $p=2$ and $J_{1}, J_{2}$ have a common endpoint. If the intervals $J_{i}$ are closed then $p=1$.

3. If $J, J^{\prime}$ are invariant non degenerate intervals, then $J \cap J^{\prime} \neq \emptyset$.

Proof.

i) If $J$ is a non degenerate interval then there exists $(x, y) \in J \times J$ such that $(x, y)$ is a Li-Yorke pair, thus $\lim \sup _{n \rightarrow+\infty}\left|f^{n}(J)\right|>0$ and for every $n \geq 0$ the interval $f^{n}(J)$ is not reduced to a point.

ii) Let $J_{1}, \ldots, J_{p}$ be disjoint non degenerate intervals such that $f\left(J_{i}\right) \subset J_{i+1} \bmod p$. Suppose that there exist $0 \leq i, j \leq p$ such that the distance $D$ between $J_{i}$ and $J_{j}$ is positive. By continuity there exists $\eta>0$ such that if $|x-y|<\eta$ then $\left|f^{k}(x)-f^{k}(y)\right|<D$ for all $0 \leq k \leq p$. If $(x, y) \in$ $J_{i} \times J_{j}$ then for all $l \geq 0$ one has $\left|f^{l p}(x)-f^{l p}(y)\right| \geq D$ thus for all $n \geq 0\left|f^{n}(x)-f^{n}(y)\right| \geq \eta$, which contradicts the assumption that $f$ is densely chaotic. If the intervals $J_{i}$ are closed it implies that $p=1$; otherwise it implies that $p=1$ or $p=2$, and if $p=2$ then $J_{1}$ and $J_{2}$ have a common endpoint.

iii) Let $J, J^{\prime}$ be two invariant non degenerate intervals. Then there exists a Li-Yorke pair $\left(x, x^{\prime}\right)$ in $J \times J^{\prime}$, in particular there exists an increasing sequence $\left(n_{i}\right)$ such that $\lim _{i \rightarrow+\infty} \mid f^{n_{i}}(x)-$ $f^{n_{i}}\left(x^{\prime}\right) \mid=0$. By compactness there exist $\left(m_{i}\right)$ a subsequence of $\left(n_{i}\right)$ and a point $z$ such that $\lim _{i \rightarrow+\infty} f^{m_{i}}(x)=\lim _{i \rightarrow+\infty} f^{m_{i}}\left(x^{\prime}\right)=z$ and the point $z$ belongs to $J \cap J^{\prime}$.

Lemma 2.2 Let $f$ be a densely chaotic interval map. Suppose that there exists a sequence of non degenerate invariant intervals $\left(J_{n}\right)_{n \geq 0}$ such that $\lim _{n \rightarrow+\infty}\left|J_{n}\right|=0$. Then there exists a point $z \in \bigcap_{n \geq 0} J_{n}$ and $f(z)=z$.

Moreover there exists a subsequence of closed non degenerate intervals $\left(J_{n}^{\prime}\right)_{n \geq 0}$ such that $f\left(J_{n}^{\prime}\right) \subset J_{n}^{\prime}, \lim _{n \rightarrow+\infty}\left|J_{n}^{\prime}\right|=0$ and for all $n \geq 0, J_{n+1}^{\prime}$ is included in the interior of $J_{n}^{\prime}$ with respect to the induced topology on $J_{0}^{\prime}$.

Proof. First we show that $\bigcap_{n=0}^{+\infty} J_{n} \neq \emptyset$. If $\bigcap_{n=0}^{N} J_{n}$ is non degenerate for all $N \geq 0$ then $\bigcap_{n=0}^{+\infty} J_{n}$ is not empty. Otherwise let $N \geq 0$ be the greatest integer such that $\bigcap_{n=0}^{N} J_{n}$ is non degenerate. The interval $K=\bigcap_{n=0}^{N} J_{n}$ is closed, non degenerate and $f(K) \subset K$. By Lemma 2.1(iii), 
$J_{N+1} \cap K \neq \emptyset$ thus the set $J_{N+1} \cap K$ is reduced to one point $z$. For every $n \geq 0$ one has $J_{n} \cap K \neq \emptyset$ and $J_{n} \cap J_{N+1} \neq \emptyset$ by Lemma 2.1(iii) thus by connectedness $z \in J_{n}$. Consequently $z \in \bigcap_{n=0}^{+\infty} J_{n}$.

The set $\bigcap_{n=0}^{+\infty} J_{n}$ is reduced to a single point $z$ because $\left|J_{n}\right| \rightarrow 0$. One has $f(z)=z$ because $f\left(J_{n}\right) \subset J_{n}$ for all $n \geq 0$.

There exists an increasing sequence $\left(n_{i}\right)_{i \geq 0}$ such that either $J_{n_{i}} \cap(z,+\infty) \neq \emptyset$ for all $i \geq 0$ or $J_{n_{i}} \cap(-\infty, z) \neq \emptyset$ for all $i \geq 0$. Define $K_{n}=\bigcap_{i=0}^{n} J_{n_{i}}$; this is a closed non degenerate interval, $f\left(K_{n}\right) \subset K_{n}$ and $K_{n+1} \subset K_{n}$.

Case 1. There exists an increasing sequence $\left(m_{i}\right)_{i \geq 0}$ such that $K_{m_{i+1}} \subset \operatorname{Int}\left(K_{m_{i}}\right)$ for all $i \geq 0$. Take then $J_{i}^{\prime}=K_{m_{i}}$.

Case 2. If the assumption of case 1 is not satisfied then there exists $N \geq 0$ such that for all $n \geq N, K_{n} \not \subset \operatorname{Int}\left(K_{N}\right)$, that is, either $\min K_{n}=\min K_{N}$ for all $n \geq N$ or $\max K_{n}=\max K_{N}$ for all $n \geq N$. Since $\left|K_{n}\right| \rightarrow 0$ one can find an increasing sequence $\left(m_{i}\right)_{i \geq 0}$ with $m_{0}=N$ such that $\left|K_{m_{i+1}}\right|<\left|K_{m_{i}}\right|$ for all $i \geq 0$. In this case $J_{i}^{\prime}=K_{m_{i}}$ is a suitable subsequence of intervals.

Lemma 2.3 Let $J$ be a bounded interval and $f: J \rightarrow J$ a continuous map with no fixed point. If $K \subset J$ is a compact interval then $\lim _{n \rightarrow+\infty}\left|f^{n}(K)\right|=0$.

Proof. Suppose that $f(x)<x$ for all $x \in J$, the case $f(x)>x$ for all $x \in J$ being similar. Write $f^{n}(K)=\left[a_{n}, b_{n}\right]$. For every $n \geq 0$ there exists $x \in\left[a_{n}, b_{n}\right]$ such that $f(x)=b_{n+1}$, thus $b_{n+1}<x \leq b_{n}$. The sequence $\left(b_{n}\right)_{n \geq 0}$ is decreasing thus has a limit in $\bar{J}$; let $z=\lim _{n \rightarrow+\infty} b_{n}$. Suppose that $z \in J$. Let $\varepsilon>0$ such that $f(z)+\varepsilon<z$. By continuity there exists $\eta>0$ such that if $|x-z|<\eta$ then $|f(x)-f(z)|<\varepsilon$. Let $n \geq 0$ such that $\left|b_{n}-z\right|<\eta$. Then for all $x \in\left[z, b_{n}\right]$ one has $f(x)<f(z)+\varepsilon<z$ and for all $x \in\left[a_{n}, z\right)$ one has $f(x)<x<z$. This implies that $b_{n+1}<z$, which is absurd. Hence $z=\inf J$ and $z<a_{n} \leq b_{n}$ for all $n \geq 0$. This implies that $\left|f^{n}(K)\right| \leq\left|b_{n}-z\right| \rightarrow 0$ when $n$ goes to infinity.

Proposition 2.4 (Snoha [16]) Let $f$ be an interval map and $\delta>0$. The following conditions are equivalent:

- $f$ is generically $\delta$-chaotic,

- for all non degenerate intervals $J_{1}, J_{2}$ one has $\lim \sup _{n \rightarrow+\infty}\left|f^{n}\left(J_{1}\right)\right|>\delta$ and $\lim \inf _{n \rightarrow+\infty} \operatorname{dist}\left(f^{n}\left(J_{1}\right), f^{n}(\right.$ 0 (where $\operatorname{dist}(\cdot, \cdot)$ denotes the distance between two sets),

Lemma 2.5 Let $f$ be a densely chaotic interval map. Suppose that there exists $\varepsilon>0$ such that, for every non degenerate invariant interval $J,|J| \geq \varepsilon$. Then $f$ is generically chaotic.

Proof. Suppose that

$$
\forall \delta>0, \exists J \text { closed non degenerate interval, } \forall n \geq 0,\left|f^{n}(J)\right| \leq \delta .
$$

We are going to show that is not possible. Let $0<\delta<\varepsilon / 4$ and let $J$ be a closed non degenerate interval such that $\left|f^{n}(J)\right| \leq \delta$ for all $n \geq 0$. There exists a Li-Yorke pair $(x, y) \in J \times J$ because $f$ is densely chaotic, thus

$$
\limsup _{n \rightarrow+\infty}\left|f^{n}(J)\right|>0 .
$$


This implies that there exist $N, p$ such that $f^{N}(J) \cap f^{N+p}(J) \neq \emptyset$, thus $f^{n}(J) \cap f^{n+p}(J) \neq \emptyset$ for all $n \geq N$. Since $f^{n}(J)$ is an interval, this implies that, for every $0 \leq i \leq p-1$, the set $Z_{i}=\bigcup_{k \geq 0} f^{N+i+k p}(J)$ is an interval, too. Consequently, the set $Z=\bigcup_{n \geq N} f^{n}(J)$ has at most $p$ connected components, which are non degenerate by Lemma 2.1(i). The image of a connected component is connected and $f\left(Z_{i}\right) \subset Z_{i+1} \bmod p$, thus the connected components of $Z$ are necessarily cyclically mapped into each other and Lemma 2.1(ii) applies: $Z$ has, either one connected component, or two connected components with a common endpoint, and $\bar{Z}$ is a closed interval.

If there exist a point $z$ and an integer $n_{0} \geq N$ such that $f^{2}(z)=z$ and $z \in f^{n_{0}}(J)$ then $z \in f^{n_{0}+2 k}(J)$ for all $k \geq 0$. Since $\left|f^{n}(J)\right| \leq \delta$ for all $n \geq 0$ one gets that $\left|\bigcup_{k \geq 0} f^{n_{0}+2 k}(J)\right| \leq 2 \delta$ and $\left|\bigcup_{k \geq 0} f^{n_{0}+2 k+1}(J)\right| \leq 2 \delta$. Let $L=\overline{\bigcup_{n \geq n_{0}} f^{n}(J)}=f^{n_{0}-N}(\bar{Z})$. Then $L$ is a closed non degenerate interval, $f(L) \subset L$ and $|L| \leq 4 \delta$. Moreover $|L| \geq \varepsilon$ according to the hypothesis of the Lemma, which is a contradiction because we have chosen $\delta<\varepsilon / 4$. We deduce that $Z$ contains no point $z$ such that $f^{2}(z)=z$.

Let $Z_{0}$ be the connected component of $Z$ containing $f^{N}(J)$ and put $g=f^{2}$. Then $g\left(Z_{0}\right) \subset Z_{0}$ and $\left.g\right|_{Z_{0}}$ has no fixed point. The interval $K=f^{N}(J)$ is compact because $J$ is compact and $f^{N}$ is continuous, so Lemma 2.3 applies and we get that $\lim _{n \rightarrow+\infty}\left|f^{N+2 n}(J)\right|=0$. By continuity of $f$ we get that $\left|f^{n}(J)\right| \rightarrow 0$ when $n$ goes to infinity, which contradicts Equation (2). We conclude that Equation (11) is false, consequently there exists $\delta>0$ such that for all closed non degenerate intervals $J, \lim _{\sup _{n \rightarrow+\infty}}\left|f^{n}(J)\right| \geq \delta$. The map $f$ is densely chaotic thus for every non degenerate intervals $J_{1}, J_{2}$ there is a Li-Yorke pair in $J_{1} \times J_{2}$, hence $\liminf \operatorname{in}_{n \rightarrow+\infty} \operatorname{dist}\left(f^{n}\left(J_{1}\right), f^{n}\left(J_{2}\right)\right)=0$. Then Proposition 2.4 implies that $f$ is generically chaotic.

\section{$3 \quad$ Structure of densely chaotic interval maps}

Recall that the interval map $g$ has a horseshoe if there exist two closed non degenerate subintervals $J, K$ with disjoint interiors such that $g(J) \cap g(K) \supset J \cup K$.

Theorem 3.1 Let $f$ be an interval map. If $f$ is densely chaotic but not generically chaotic then there exists a sequence of invariant non degenerate subintervals $\left(J_{n}\right)_{n \geq 0}$ such that $J_{n+1} \subset J_{n}$, $\lim _{n \rightarrow+\infty}\left|J_{n}\right|=0$, and $\left.f^{2}\right|_{J_{n}}$ has a horseshoe for all $n \geq 0$.

We need two lemmas in the proof of this theorem. Lemma 3.2 is proven in 9 under slightly weaker hypotheses, see also [4, p 28]. Lemma 3.3 can be found in [4, p31].

Lemma 3.2 Let $f$ be an interval map with no horseshoe and $x$ a point. Write $x_{n}=f^{n}(x)$ for all $n \geq 0$. Suppose that $x_{n+1} \geq x_{n}$ and $x_{m+1} \leq x_{m}$. Then $x_{n} \leq x_{m}$.

Lemma 3.3 Let $f$ be an interval map such that $f^{2}$ has no horseshoe. Let $x$ be a point which is not ultimately periodic and write $x_{n}=f^{n}(x)$ for $n \geq 0$. Suppose that there exists $k_{0} \geq 2$ such that either $x_{k_{0}}<x_{0}<x_{1}$ or $x_{k_{0}}>x_{0}>x_{1}$. Then there exist a fixed point $z$ and an integer $N$ such that, for all $n \geq N, x_{n}>z \Leftrightarrow x_{n+1}<z$.

Proof of Theorem 3.1. By assumption the map $f$ is not generically chaotic thus, by Lemma 2.5.

$\forall \varepsilon>0$, there exists an invariant non degenerate interval $J$ such that $|J|<\varepsilon$.

Let $\left(I_{n}\right)_{n \geq 0}$ be a sequence of invariant non degenerate intervals $I_{n}$ such that $\left|I_{n}\right| \rightarrow 0$. By Lemma 2.2 there exists a sequence of invariant non degenerate intervals $\left(J_{n}\right)_{n \geq 0}$ such that 
$\lim _{n \rightarrow+\infty}\left|J_{n}\right|=0$, and $J_{n+1} \subset \operatorname{Int}\left(J_{n}\right)$ with respect to the induced topology on $J_{0}$. From now on we fix $n_{0} \geq 0$ and we restrict to the interval $J_{n_{0}}$. The map $\left.f\right|_{J_{n_{0}}}$ is densely chaotic, the set $\bigcap_{n \geq n_{0}} J_{n}$ is reduced to a single point $z$ and $f(z)=z$. Let

$$
\mathcal{P}=\left\{x \in J_{n_{0}} \mid \exists p \geq 1, \lim _{n \rightarrow+\infty} f^{n p}(x) \text { exists }\right\} .
$$

If $x, y \in \mathcal{P}$ then $(x, y)$ is not a Li-Yorke pair, thus the set $J_{n_{0}} \backslash \mathcal{P}$ is not empty.

Assume that $\left.f^{2}\right|_{J_{n_{0}}}$ has no horseshoe; we are going to prove that this is absurd. Let $x_{0} \in$ $J_{n_{0}} \backslash \mathcal{P}$ and write $x_{n}=f^{n}\left(x_{0}\right)$ for all $n \geq 0$. According to Lemma 3.3 there exist a fixed point $c$ and an integer $N$ such that, for all $n \geq 0, x_{N+2 n}<c<x_{N+2 n+1}$. Suppose for instance that $c \leq z$, the case with reverse inequality being symmetric. Since $x_{0} \notin \mathcal{P}$ the sequence $\left(x_{N+2 n}\right)_{n \geq 0}$ is not ultimately monotone, thus there exists $i \geq 0$ such that

$$
x_{N+2 i+2}<x_{N+2 i}<c \leq z .
$$

By continuity there exists a closed non degenerate interval $K$ containing $x_{N+2 i}$ such that $z \notin K$ and for all $y \in K, f^{2}(y)<y$. Let $k \geq n_{0}$ such that $K<J_{k}$.

The set $K \times K$ contains a Li-Yorke pair because $f$ is densely chaotic, thus $\lim \sup _{n \rightarrow+\infty}\left|f^{n}(K)\right|>$ 0 and there exist $p, q$ such that $f^{q+p}(K) \cap f^{q}(K) \neq \emptyset$. Let $L=\overline{\bigcup_{n \geq q} f^{n}(K)}$. One has $f(L) \subset L$. The same argument as for $\bar{Z}$ in the proof of Lemma 2.5 implies that $L$ is an invariant non degenerate interval. Moreover Lemma 2.1(iii) implies that $L \cap J_{n} \neq \emptyset$ for all $n \geq n_{0}$. Since $J_{k+1} \subset \operatorname{Int}\left(J_{k}\right)$, this implies that there exists an integer $n \geq 0$ such that $f^{n}(K) \cap \operatorname{Int}\left(J_{k}\right) \neq \emptyset$, thus there exists a closed non degenerate subinterval $K^{\prime} \subset K$ such that $f^{n}\left(K^{\prime}\right) \subset J_{k}$.

Let $m_{0} \geq n / 2$ and $g=f^{2}$. For all $y \in K^{\prime}$ and all $m \geq m_{0}$ one has $g^{m}(y) \in J_{k}$ because $f\left(J_{k}\right) \subset J_{k}$, thus

$$
g(y)<y<g^{m}(y)
$$

This implies that there exists $0<j<m_{0}$ such that $g^{j}(y)<g^{j+1}(y)$. By assumption $g$ has no horseshoe thus $g^{j}(y) \leq y$ by Lemma 3.2. For all $m \geq m_{0}$, one has $y \leq g^{m}(y)$, thus the same lemma implies that $g^{m+1}(y) \leq g^{m}(y)$. Consequently, $\left(g^{m}(y)\right)_{m \geq m_{0}}$ is a non increasing sequence, thus it converges. But this implies that $K^{\prime} \times K^{\prime}$ contains no Li-Yorke pair, which contradicts the fact that $f$ is densely chaotic. This concludes the proof.

Next theorem sums up two results on horseshoes, the first point is due to Block and Coppel [3], the second one derives from [2] (see also [4, p 196]).

Theorem 3.4 Let $f$ be an interval map with a horseshoe. Then

- $f$ is of type 3 for Sharkovskiu's order,

- $h_{t o p}(f) \geq \log 2$.

According to Theorem 1.5, a generically chaotic interval map $f$ admits a transitive subinterval, thus next theorem implies that $f^{2}$ has a horseshoe.

Theorem 3.5 (Block-Coven [1]) Let $f$ be a transitive interval map. Then $f^{2}$ has a horseshoe.

Corollary 3.6 Let $f$ be a densely chaotic interval map. Then $f^{2}$ has a horseshoe, $h_{\text {top }}(f) \geq$ $\frac{\log 2}{2}$ and $f$ is of type at most 6 for Sharkovskiu's order. 
Proof. If $f$ is generically chaotic then $f^{2}$ has a horseshoe by Theorems 1.5 and 3.5 , otherwise $f^{2}$ has a horseshoe by Theorem 3.1. By Theorem 3.4. $h_{t o p}(f) \geq \frac{\log 2}{2}$ and $f^{2}$ has a periodic point of period 3 , thus $f$ has a periodic point of period 3 or 6 .

Example 3.7 In Corollary 3.6 equalities are possible. Consider the "square-root" of the tent map, pictured on Figure 1. The map $g$ swaps the intervals $[0,1 / 2]$ and $[1 / 2,1]$ thus every periodic point $x \neq 1 / 2$ has an even period. Moreover the intervals $[0,1 / 4],[1 / 4,1 / 2]$ form a horseshoe for $g^{2}$, so Theorem 3.4 implies that $g$ is of type 6 for Sharkovski1's order. The map $g^{2}$ restricted to either $[0,1 / 2]$ or $[1 / 2,1]$ is the classical tent map (upside down on $[0,1 / 2]$ ), which is known to be mixing (see, e.g., [4, p 159]), so $g$ is transitive (thus generically chaotic). Finally the topological entropy of $g^{2}$ is equal to $\log 2$ (use either the fact that it is Markov or the combination of Theorem 3.4 and [6, Proposition (14.20)]), hence $h_{t o p}(g)=\frac{\log 2}{2}$.

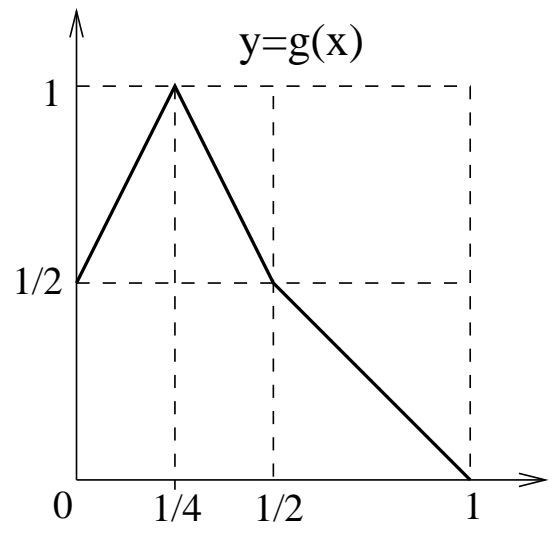

Figure 1: Densely chaotic map of entropy $\frac{\log 2}{2}$ and type 6 .

This example shows that the infimum of the topological entropy of densely (respectively generically) chaotic interval maps is reached and is equal to $\frac{\log 2}{2}$.

There also exist transitive (thus generically chaotic) interval maps of type $2 k+1$ for all $k \geq 1$ [1]. It derives from [2] that the topological entropy of a map of type $2 k+1$ is greater than $\frac{\log 2}{2}$.

\section{References}

\section{References}

[1] L. Block and E. M. Coven, Topological conjugacy and transitivity for a class of piecewise monotone maps of the interval, Trans. Amer. Math. Soc. 300 (1987), no. 1, 297-306. MR $88 \mathrm{c}: 58032$

[2] L. Block, J. Guckenheimer, M. Misiurewicz, and L. S. Young, Periodic points and topological entropy of one dimensional maps, Global Theory of Dynamical Systems, Lecture Notes in Mathematics, no. 819, Springer-Verlag, 1980, pp. 18-34.

[3] L. S. Block and W. A. Coppel, Stratification of continuous maps of an interval, Trans. Amer. Math. Soc. 297 (1986), no. 2, 587-604. MR 88a:58164

[4] L. S. Block and W. A. Coppel, Dynamics in one dimension, Lecture Notes in Mathematics, no. 1513, Springer-Verlag, 1992. 
[5] G. J. Butler and G. Pianigiani, Periodic points and chaotic functions in the unit interval, Bull. Austral. Math. Soc. 18 (1978), no. 2, 255-265, MR58 \#13203

[6] M. Denker, C. Grillenberger, and K. Sigmund, Ergodic theory on compact spaces, Lecture Notes in Mathematics, no. 527, Springer-Verlag, 1976.

[7] W. Huang and X. Ye, Devaney's chaos or 2-scattering implies Li-Yorke's chaos, Preprint.

[8] M. Kuchta and J. Smítal, Two-point scrambled set implies chaos, European Conference on Iteration Theory (Caldes de Malavella, 1987), World Sci. Publishing, Teaneck, NJ, 1989, pp. 427-430. MR 91j:58112

[9] T. Y. Li, M. Misiurewicz, G. Pianigiani, and J. A. Yorke, No division implies chaos, Trans. Amer. Math. Soc. 273 (1982), no. 1, 191-199. MR 83i:28024

[10] T. Y. Li and J. A. Yorke, Period three implies chaos, Amer. Math. Monthly 82 (1975), no. $10,985-992$. MR $52 \# 5898$

[11] E. Murinová, Generic chaos in metric spaces, Acta Univ. M. Belii Math. 8 (2000), 43-50.

[12] J. Oxtoby, Measure and category, Springer-Verlag, 1980.

[13] J. Piórek, On the generic chaos in dynamical systems, Univ. Iagel. Acta Math. 25 (1985), 293-298. MR 87h:58131

[14] A. N. Sharkovskiŭ, Co-existence of cycles of a continuous mapping of the line into itself (Russian), Ukrain. Mat. Z̆. 16 (1964), 61-71. MR 28 \#3121. English translation: Internat. J. Bifur. Chaos Appl. Sci. Engrg. 5 (1995), no. 5, 1263-1273.

[15] J. Smítal, Chaotic functions with zero topological entropy, Trans. Amer. Math. Soc. 297 (1986), no. 1, 269-282. MR 87m:58107

[16] L. Snoha, Generic chaos, Comment. Math. Univ. Carolin. 31 (1990), no. 4, 793-810. MR 92b:58149

[17] L'. Snoha, Dense chaos, Comment. Math. Univ. Carolin. 33 (1992), no. 4, 747-752. MR 94i: 58128

[18] P. Štefan, A theorem of Šarkovskii on the existence of periodic orbits of continuous endomorphisms of the real line, Comm. Math. Phys. 54 (1977), no. 3, 237-248. MR 56 \#3894

Laboratoire de Mathématiques - Topologie et Dynamique - Bâtiment 425 - Université ParisSud - 91405 Orsay cedex - France e-mail : sylvie.ruette@math.u-psud.fr 